Abb. 1 Agnus castus, der Mönchspfeffer, harmonisiert das Zusammenspiel zwischen Hypophyse und Ovar. (c) tunedin/Adobe Stock

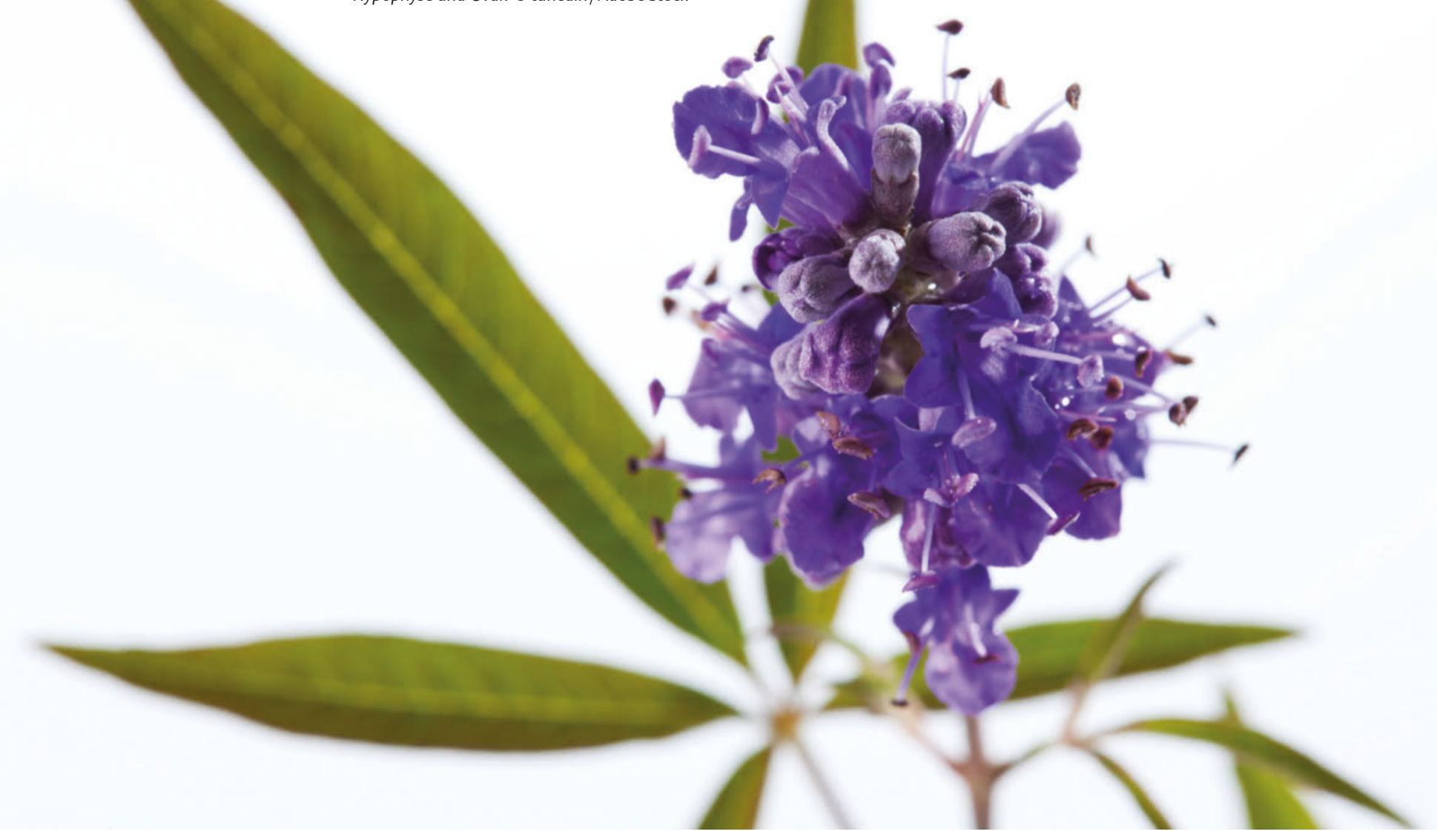

Fertilitätsstörungen

\title{
Therapieansätze der anthroposophischen Medizin
}

Das ärztliche Gespräch, anthroposophische Arzneimittel, nicht medikamentöse Verfahren Die anthroposophische Medizin richtet den Blick auf den ganzen Menschen: somatische Aspekte, die Biographie, den lebendigen Leib und das Empfindungsleben

Gabriela Stammer

\section{Einleitung}

Insgesamt $20 \%$ aller Frauen in Deutschland sind ungewollt kinderlos. Laut statistischem Bundesamt ist die Anzahl der Geburten in den letzten 8 Jahren zwar stabil geblieben, die Quote der ungewollt kinderlosen Akademikerinnen hat sich demnach sogar von 30 auf $27 \%$ verbessert. Trotzdem scheint es schwieriger zu werden, auf natürlichem Wege schwanger zu werden.

2015 kamen in Deutschland 20000 Kinder mithilfe einer künstlichen Befruchtung zur Welt. Diese Zahl ist bemerkenswert auch angesichts der Tatsache, dass das erste IVF-Baby in diesem Jahr 40 Jahre alt geworden ist. Damals war diese Nachricht fast beängstigend und auch in den darauffolgenden Jahren hielten Paare es eher geheim, wenn ihr Kind mithilfe einer künstlichen Befruchtung gezeugt wurde. Inzwischen ist diese Option bei ungewollter Kinderlosigkeit gesellschaftlich akzeptiert. Dennoch führt nur jeder 10. Zyklus einer künstlichen Befruchtung zur Geburt eines Babys.
Der folgende Artikel legt das Augenmerk auf die therapeutischen Ansätze der Anthroposophischen Medizin bei ungewollter Kinderlosigkeit. Dabei spielen nicht nur die somatischen Aspekte eine Rolle, sondern der Blick wird insbesondere auf die Biographie, den ganzen Menschen als lebendigen Leib, sein Empfindungsleben sowie seine Individualität gelenkt. 


\section{Zusammenfassung}

In der Anthroposophischen Medizin stehen vier Behandlungselemente im Vordergrund: das ärztliche Gespräch, Arzneimittel der Anthroposophischen Medizin, nichtmedikamentöse Heilmittel wie Eurythmie oder Kunsttherapie sowie äußere Anwendungen. Dabei werden auch biographische Aspekte und das Empfindungsleben in der Therapie berücksichtigt. Damit kann die konventionelle Therapie bei Fertilitätsstörungen sinnvoll erweitert werden.

Der Artikel fokussiert das therapeutische Vorgehen bei Corpus-luteum-Schwäche, polyzystischem Ovar und Prolactinämie.

\section{Anthroposophische Medizin}

Die Besonderheiten der Anthroposophischen Medizin wurden in dieser Zeitschrift bereits ausführlich beschrieben [4], deshalb sei hier nur eine kurze Zusammenfassung wiedergegeben.

Zum Menschen als Abbild der Welt gehört nicht nur der lebendige Körper, den wir anfassen und messen können. Den rein physischen Körper haben wir mit der Mineralwelt gemein, den lebendigen Leib mit der Pflanzenwelt. Hingegen hat unser Seelenleben eine unmittelbare Wirkung auf den lebendigen Körper, wie wir im Schreck oder in der Verliebtheit selbst erleben können. Hormone wirken bis in diese Region, man denke nur an einen Menschen mit einer Hyperthyreose oder in der 1. Zyklushälfte. In die Welt des Seelenlebens gehören auch Instinkte und Triebe, das haben wir mit der Tierwelt gemein. Dem Menschen eigen hingegen ist die Fähigkeit zum Abwägen, zum Urteil, zur moralischen Haltung, zur Biographie, zur Freiheit.

In der Anthroposophischen Medizin stehen die folgenden vier Behandlungselemente im Vordergrund:

\section{Gespräch}

Das ärztliche Gespräch steht in der Anthroposophischen Medizin im Zentrum der therapeutischen Beziehung. Es werden die Beschwerden bewusst mit Fragen aus der Anthroposophischen Medizin verknüpft, z.B. wo Heilbedarf liegt und therapeutische Möglichkeiten zu suchen sind. Die Biographie und Lebensrhythmen spielen eine große Rolle.

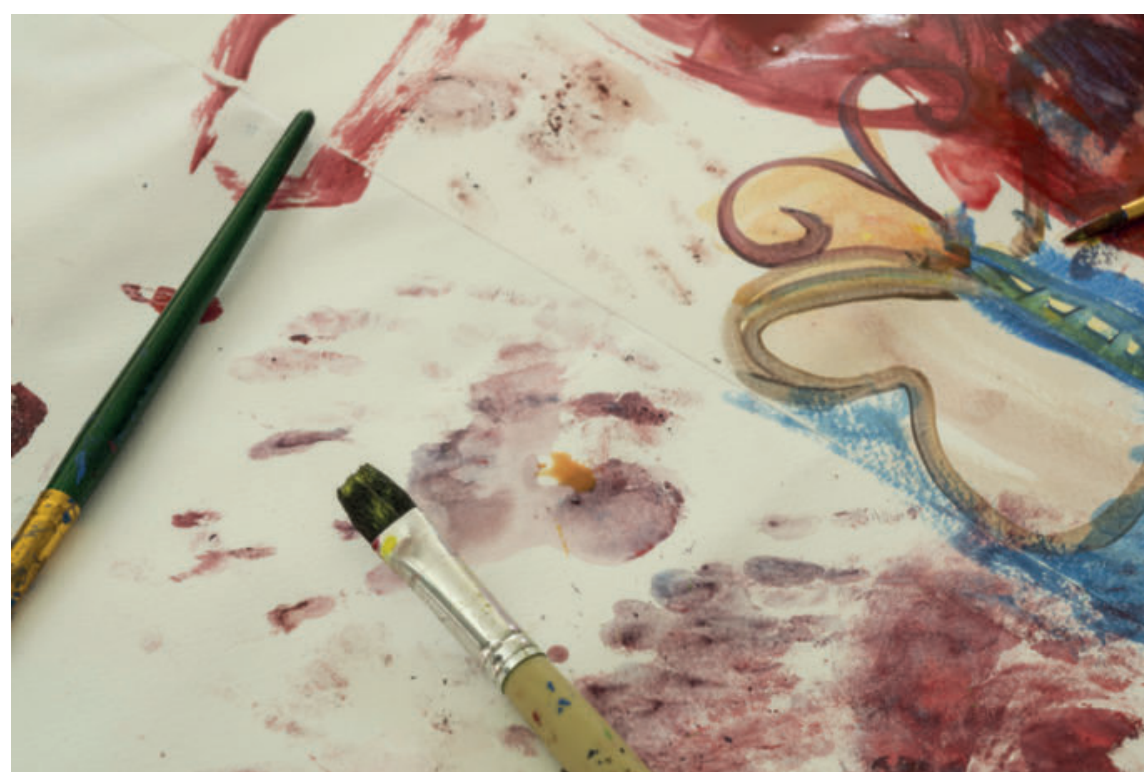

Abb. 2 Die Kunsttherapie kann neue Wege zu eigenen Ressourcen eröffnen. @ Sasipixel/Adobe Stock

\section{Medikamente der Anthroposophischen} Medizin

Die anthroposophische Arzneimitteltherapie bildet ein weiteres wesentliches Standbein. Durch Verwendung von mineralischen und pflanzlichen Substanzen in besonders verarbeiteter Form sowie potenzierten Organpräparaten aus der Tierwelt wird versucht, die vier Ebenen im Menschen anzusprechen.

Das Vademecum Anthroposophische Arzneimittel [2] ist inzwischen in der 4. Auflage erschienen und hat eine Vielzahl an Erfahrungen anthroposophisch tätiger Ärztinnen und Ärzte gesammelt. Es umfasst jetzt auch eine systematische Aufarbeitung der Erfahrungen mit Misteltherapie.

\section{Weitere nichtmedikamentöse Heilmittel} Zu den Heilmitteln zählen Eurythmietherapie, Kunsttherapie, Sprachgestaltung, rhythmische Massagen, Musiktherapie. Es gibt sehr unterschiedliche Indikationen für die Therapien. Wesentlich für diese Therapien ist eine nicht-kognitive Förderung der Fähigkeiten und der Revitalisierung sowie der Ausgleich von übermäßiger seelischer Agilität und Erschöpfung zur Entwicklung der eigenen Resilienzen.

\section{Äußere Anwendungen}

Wickel, Auflagen, Fußbäder, Dampfbäder, Öldispersionsbäder u.a. sind von hoher Wirksamkeit und unmittelbar erlebbar in ihren Wirkungen.

\section{Der weibliche Zyklus}

Der weibliche Zyklus differenziert sich in 2 Phasen. Die erste Phase ist östrogenbetont sowie durch Wachstum und Überschwang gekennzeichnet. Es herrscht eine euphorische und extrovertierte Verfassung vor. Die zweite Zyklushälfte wird vom Gelbkörperhormon geprägt: Die Schleimhaut der Gebärmutter wird verdichtet, Spiralarterien sprossen ein, die Stimmung ist eher introvertiert, manchmal zweifelnd, eher erschöpft. Wie in einer Sinuskurve ,atmet“ die weibliche Individualität in diesem monatlichen Rhythmus. Die erste Zyklushälfte (ZH) hat eine zentripetale Geste, kraftvoll, schier unbegrenzt. Die zweite Zyklushälfte hingegen ist nicht nur durch die stärkere Inkarnierung, sondern auch durch größere Wachheit und Klarheit gekennzeichnet [1].

\section{Diagnostik und Therapie}

So zahlreich wie die Gründe sind auch die Therapien für einen unerfüllten Kinderwunsch. Schon allein die organischen Ursachen sind vielfältig und bedürfen eines guten Verständnisses des weiblichen Zyklus. Mögliche Ursachen, die im Folgenden mit ihren anthroposophischen Behandlungsoptionen vorgestellt werden sollen, sind:

- Corpus-luteum-Schwäche

- Polyzystisches Ovar (PCOS)

- Prolactinämie

- Schilddrüsenfunktionsstörungen

- Verwachsungen 


\section{Zyklustempostörungen/Corpus-luteum- Insuffizienz}

Bei einer Corpus-luteum-Schwäche können die nötigen 14 Tage der zweiten Zyklushälfte nicht durchgehalten werden, d.h. der Zyklus bricht zu früh ab.

Die einfache Corpus-luteum-Insuffizienz ist häufig gut mit einem alkoholischen Auszug von Mönchspfeffer (Agnus Castus, Fertigpräparat z. B. Agnolyt ${ }^{\circledR}$ ) behandelbar. Die morgendliche Einnahme in heißer Flüssigkeit muss konsequent kontinuierlich sein. Ein messbarer Wirkungseintritt ist frühestens nach 3 Monaten zu erwarten, da die heutige Maßnahme auf denjenigen Follikel Einfluss nimmt, der in 3 Monaten reifen und ovulieren wird. Bei fehlender Wirkung der Stabilisierung ist Melissa/Phosphorus comp. (Fa. Weleda) morgens 10 Tropfen [2] zusätzlich zu Agnolyt ${ }^{\circledR}$ in der 2. Zyklushälfte genommen, ausgesprochen hilfreich. In jedem Fall ist es sehr ratsam, ein Augenmerk auf den Tagesrhythmus zu richten sowie auf regelmäßige Bewegung, z. B. tägliches Laufen. Die Ovarien sind ein Organ des Stoffwechsel-Menschen, sie sind auf einen (fast) regelmäßigen Rhythmus des Tages und ihre gute Funktionalität auf Bewegung und Durchblutung angewiesen.

\section{Behandlungsstrategien}

Gespräch: Eine zentrale Frage könnte sein, ob es im Alltag der Frau tatsächlich genügend Erlaubnis gibt für die mehr innerliche, etwas verlangsamte Phase der 2. Zyklushälfte. Gelingt es, den Raum zu schaffen, „das Bett zu bereiten“ oder gehen das Leben, der Stress, das Durchhalten, das Rauchen (,ich höre bestimmt mit dem Rauchen auf, wenn ich schwanger bin“) wie immer weiter?

Medikation: Agnolyt ${ }^{\circledR}$ Dil morgens $40 \mathrm{Tr}$., Melissa/Phosphorus comp. ${ }^{\circledR}$ Dil morgens 10 Tr. In der 2. Zyklushälfte

\section{Ratio zu Melissa/Phosphorus comp}

- Agnus castus harmonisiert das Zusammenspiel zwischen Hypophyse und Ovar. Agnus castus wirkt in tiefer Potenz (D2) ähnlich wie der originäre Pflanzenextrakt.

- Corpus luteum bovis D4: wie ein Vorbild kann dieser Anteil des Präparats eine Unterstützung zur gesunden und zuverlässigen Arbeit des Corpus luteum sein.

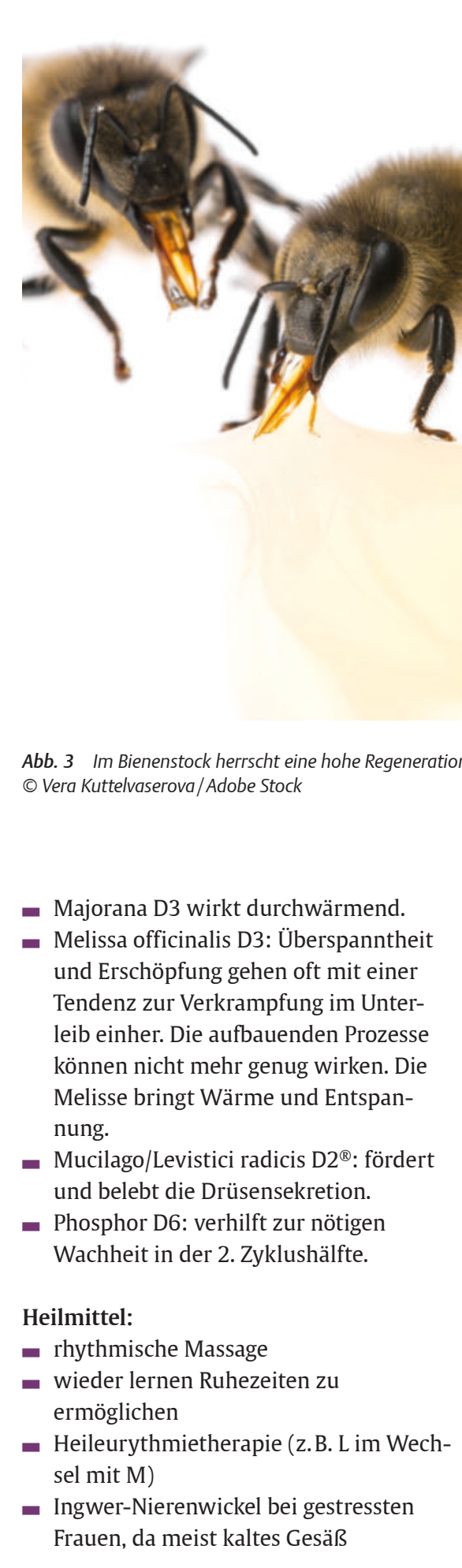

\section{Polyzystisches Ovarsyndrom}

Das polyzystische Ovarsyndrom (PCOS) hat unterschiedliche Erscheinungsformen. Zum einen findet man einen im Ultraschall vergleichbaren Befund häufig nach Absetzen der Pille in einer oftmals über Monate dauernden Phase der Regeneration der Ovarien. Bei Persistieren (nach 6 Monaten) oder Ungeduld (auch sofort nach Absetzen der Pille) kann das oben genannte Konzept auch hier angewendet werden (Agnolyt ${ }^{\circledR}$ Dil morgens 40 Tr., Melissa/Phosphorus comp. Dil morgens $10 \mathrm{Tr}$. In der 2. Zyklushälfte).

\section{PCOS bei adipösen Frauen}

Neben genetischen und familiären Syndromen polyzystischer Ovarien (Prägung intrauterin durch zu hohe Anti-Müller-Hormonspiegel der Mutter) ist dieses Phänomen zunehmend bei übergewichtigen Patientinnen zu beobachten. Im Rahmen eines metabolischen Syndroms kommt es durch eine Insulinresistenz zu einer Blockade der Ovarien. Dies wiederum bewirkt eine Hyperandrogenämie. Hierdurch kommt es mangels verringerter Glykolyse zur vermehrten Fettspeicherung, die das Problem erneut stützt: ein Teufelskreis.

Auch eine primär vorliegende Hyperandrogenämie kann die Insulinresistenz und Hyperinsulinämie induzieren. Hierbei handelt es sich um ein 21-Hydroxylasemangel vom Typ des „Late-onset“-AGS mit polyzystischen Ovarien.

Hin und wieder sollte man auch den Gebrauch von Anabolika erfragen.

\section{Behandlungsstrategien}

Gespräch: Der Rhythmus des Monatszyklus ist wie angehalten und steht auf der Stelle. 
Hier ist ein Beratungsgespräch nötig: Schon $5 \mathrm{~kg}$ Gewichtsabnahme schaffen in $80 \%$ der adipositasbedingten Fälle einen Start in den Zyklus. Tägliches Walken oder Joggen fördert die fließende Lebendigkeit in der wie starr gewordenen leiblichen Tätigkeit. Das rhythmische Laufen sowie das Auftreten auf die Erde haben eine kräftigende Wirkung, anders als Fahrradfahren oder Schwimmen. Medikation: Agnolyt ${ }^{\circledR}$ Tr., Ovaria comp. ${ }^{\circledR}$ Ampullen 2- bis 3-mal wöchentlich s.c. gespritzt (notfalls oral, unter der Zunge stehen lassen)

\section{Ratio zu Ovaria comp.}

- Apis regina D5: Im Bienenstock ist immer konstante Wärme und Bewegung. Es herrscht eine hohe Regenerationskraft.

- Argentum met. D5: Silber gehört bei den Metallen zu den Vertretern der aufbauenden, regenerativen Substanzen.

- Ovaria bovis D7 ${ }^{\circledR}$ (Wala): Wie urbildhaft wird an die originäre Tätigkeit „erinnert".

\section{Heilmittel:}

- Heileurythmietherapie (z. B. O-Übung)

- Kunsttherapie: Plastizieren, ggf. Malen

\section{PCOS bei schlanken Frauen}

Bei Patientinnen mit PCOS und überschlanker Konstitution bedarf es vor allem einer großen Portion Geduld. Es handelt sich oft um eher willens- und leistungsbetonte Frauen. Ihr eigenes Frauenbild ist oft nicht klar und wird nicht gern ausgefüllt. Die Übergänge zur Anorexie können fließend sein. Therapeutisch braucht man hin und wieder mehrere Jahre. Agnolyt ${ }^{\circledR}$ Dil morgens 40 Tr., Ovaria comp. Ampullen im Wechsel mit Hypophysis Gl ${ }^{\circledR}$ D6 haben sich sehr bewährt. Die Patientinnen geben oft selbst an, mit welchem der Medikamente sie besser gefördert sind. Die Therapie kann immer wieder angepasst und variiert werden.

Bei einem „dringendem Kinderwunsch“ findet man allerdings in der Anthroposophischen Medizin keine rasche Antwort, die Therapie braucht Geduld.

\section{Behandlungsstrategien}

Gespräch: Im Gespräch gilt es herauszuarbeiten, welche Erfahrungen in der Biographie zu den Mustern der "Leistungstochter“ geführt haben. Oft steht ein Verlust des Urvertrauens um das 9. Lebensjahr (Rubikon) dahinter. In dieser Lebensphase fragt der junge Mensch nach seiner Einmaligkeit und Besonderheit und er entdeckt die Bedeutung des Todes, die große Sorge vor dem Alleinsein nachdem die Eltern versterben könnten. Offen gebliebene Fragen, z.B. nach dem Tod einer Bezugsperson, haben oft Nachwirkungen weit in die Biographie hinein.

Medikation: Agnolyt ${ }^{\circledR}$ Dil morgens 40 Tr., Ovaria comp. Ampullen 2- bis 3-mal wöchentlich s.c. gespritzt im Wechsel mit Hypophysis Gl D6 2- bis 3-mal wöchentlich s.c. gespritzt (notfalls oral, unter der Zunge stehen lassen).

\section{Heilmittel:}

- Heileurythmietherapie (z. B. E-Übung)

- Rhythmische Massage (Durchwärmung, Entspannung, Vertrauen)

- Sprachgestaltung (auch sehr wirksam: Atem-, Sprech- und Stimmtherapie nach Schlaffhorst-Andersen)

\section{Prolactinom}

Die Prolactinämie kann mit oder ohne nennenswertes Hypophysenadenom einhergehen. Bei nachgewiesenem Prolactinom und guter Operabilität bzw. drohender Schädigung des Chiasma opticus sollte eine Operation in Erwägung gezogen werden. Bei Werten unter $100 \mu \mathrm{g} / \mathrm{ml}$ und fehlender OP-Indikation kann ein Therapieversuch mit Agnolyt ${ }^{\circledR}$ Dil. Morgens 40 Tr. sehr hilfreich sein, da der Wirkmechanismus von Agnus castus auch über die Senkung des Prolactin verläuft. Dies ist auch der Grund für die 
Kontraindikationen von Agnus castus: Stillen und Antipsychotika mit begleitender Hyperprolactinämie. Hier würde Agnus castus die antipsychotische Komponente der Medikamente auch reduzieren, was zu erneuten Schüben der Psychose führen kann.

\section{Schilddrüsenerkrankungen}

Die Schilddrüse hat mit einer Über- oder Unterfunktion Einfluss auf die Funktion der Ovarien. Eine suffiziente Einstellung auf Normwerte ist ratsam. Es gibt aber auch Schwangerschaften bei pathologischen Schilddrüsenwerten.

Die Schilddrüse ist ein bemerkenswertes Organ, dessen therapeutische Begleitung sich nicht nur auf ein TSH von unter 2,1 mlU/1 beschränkt. Eine Therapie aus der Anthroposophischen Medizin kann bei allen Schilddrüsenerkrankungen eine sehr hilfreiche Unterstützung sein [3]. Für die Autorin ist es ein großes Geschenk, dass eine intensive Zusammenarbeit besteht mit mehreren Allgemeinmedizinern in Praxisgemeinschaft, die die Patientinnen in diesem Sinne suffizient mitbetreuen.

\section{Verwachsungen}

Bei Verwachsungen kann die Befruchtung mechanisch durch eine vorhandene Barriere verhindert werden. Besteht der Verdacht, dass die Konzeption durch Adhäsionen behindert ist, z. B. bei Z.n. früheren Bauchoperationen, oder eine Endometriose vorliegt, sollte die diagnostische bzw. zeitgleich therapeutische Laparoskopie angestrebt werden. In gleicher OP sollte die Durchgängigkeit der Eileiter überprüft werden.

Bei Verwachsungen als Hinderungsgrund (z.B. große Segel mit vollständiger Trennung von Fimbrientrichter und Ovar, z. B. nach Chlamydieninfektion) hilft nur eine mechanische Lösung.

\section{Oligo- und Asthenozoospermie der werdenden Väter}

Immer mehr Männer sind eingeschränkt oder gar nicht zeugungsfähig. Zu vermuten ist hier die östrogenartige Wirkung diverser Umweltgifte und Weichmacher.

Ein Therapieversuch mit Testes comp ${ }^{\circledR}$ Glob ist ratsam und immer wieder hilfreich.

\section{Behandlungsstrategien}

Medikation: Testes comp, Glob (abends 10 Glob.)

Ratio zu Testes comp.

- Apis regina D5: Im Bienenstock ist immer konstante Wärme und Bewegung. Es herrscht eine hohe Regenerationskraft.

- Argentum met. D5: Silber gehört bei den Metallen zu den Vertretern der aufbauenden, regenerativen Substanzen.

- Testes bovis D4: Wie urbildhaft wird an die originäre Tätigkeit „erinnert“.

\section{Funktionelle Fertilitätsstörungen}

Die funktionellen Fertilitätsstörungen sind in der therapeutischen Begleitung eine große Herausforderung. Meist wurde alles Erdenkliche untersucht, auch invasiv abgeklärt und keine Ursache gefunden, warum sich die gewünschte Schwangerschaft nicht einstellen will. Mehrere IVF- oder ICSI-Zyklen haben nicht zur Schwangerschaft geführt.

Hier gilt es zunächst, ein biographisches Verständnis für die Situation zu entwickeln. Einige der genannten Heilmittel können auch in dieser Situation als Hilfestellung zum Einsatz kommen. IVF und ICSI sind physisch und emotional anstrengend, regenerative Therapien, z. B. mit Aurum/Apis regina comp. Glob (morgens 10 Globuli) helfen auch der erschöpften und traurigen Seele. Letztlich ist es aber die Aufgabe, den werdenden Eltern (meist den werdenden Müttern) zu raten, den Fokus der Aufmerksamkeit weg vom Augenblick der Befruchtung zu nehmen. Aber wie kann man das? Wie kann man bewusst nicht daran denken? Hier braucht es Gespräche, die vermitteln helfen, dass wir an der Schwelle des Gezeugt- und Geborenwerdens, wie auch an der Schwelle des Sterbens, nicht mehr mit unserem normalen Willen hinreichen. Es muss schon jemand die befruchtete Eizelle haben wollen, sie ergreifen. Es gilt eine Haltung des Empfangens zu üben, Empfangen ist allerdings fast das Gegenteil von Nehmen. Es scheint notwendig, den Respekt für die Freiheit des Kindes auf der uns unbekannten anderen Seite zu üben.

\section{Fazit}

Die Rate der ungewollt kinderlosen Paare ist hoch. Es liegen unterschiedlichste Gründe und Diagnosen dazu vor. In dieser schwierigen Lebenssituation ist eine Therapie mit Gesprächen, Medikamenten und Heilmitteln der Anthroposophischen Medizin sehr hilfreich.

Interessenkonflikt: Die Autorin erklärt, dass keine wirtschaftlichen oder persönlichen Verbindungen bestehen.

Online zu finden unter

http://dx.doi.org/10.1055/a-0821-8141

\section{Literatur \\ $\overline{1}$ Maris B. Frauenheilkunde und Geburtshilfe. Berlin: Salumed; 2012 \\ 2 GAÄD. Vademecum Anthroposophische Arznei- mittel. 4. Aufl. Filderstadt: GAÄD; 2017 \\ 3 Girke M. Innere Medizin. 2. Aufl. Berlin: Salu- med; 2012 \\ 4 Stammer G. Behandlung gynäkologischer Er- krankungen mit anthroposophischer Medizin. Z Komplementmed 2017; 6: 30-35}

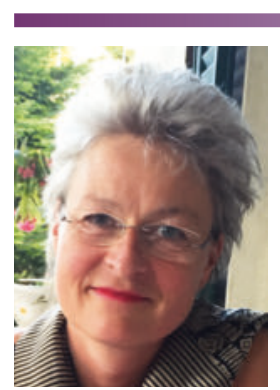

Dr. med. Gabriela Stammer

Ärztin für Frauenheilkunde und Anthroposophische Medizin

(GÄ̈D)

Klosteramthof 1

30974 Wennigsen

gabriela-stammer@t-online.de

Gabriela Stammer arbeitet seit 1998 als niedergelassene Frauenärztin in Wennigsen. Seit 2008 gehört sie zum erweiterten und seit 2013 zum geschäftsführenden Vorstand der Gesellschaft Anthroposophischer Ärzte in Deutschland (GAÄD). 